\title{
Chronic Hyperplastic and Erythematous Candidiasis Induced by Ill-fitting Complete Denture: A Case Report
}

\author{
Shimiyan Debbarma ${ }^{1}$, Anshuman Jamdade ${ }^{2}$, Satyapal Yadav ${ }^{3}$, Neeraj K Yadav ${ }^{4}$
}

\begin{abstract}
Oral candidiasis is one of the common fungal infections, affecting the various parts of oral mucosa which is caused by Candida albicans. The pathogenicity of Candida is facilitated by many virulence factors, some of which are those for an observance to host tissues and medical devices and secretion of hydrolytic enzymes. Valuation of predisposing factors plays an important role in the treatment of candidal infection. Carefully recording medical history plays a vital role. Topical antifungal application is suggested as a first-line treatment for simple oral candidiasis. Especially in denture wearing patients, it is a common inflammatory reaction, multifactorial etiology, which is usually accompanying by Candida species, particularly C. albicans, because of its high virulence, the capability to adhere and form biofilms on oral cavity mucosa and denture surfaces. This article intensifies the management of oral candidiasis associated with ill-fitting denture commonly encountered in day-to-day dental practice. Keywords: Antifungal, Candida albicans, Hyperplastic candidiasis, III-fitting complete denture, Predisposing factors. Journal of Mahatma Gandhi University of Medical Sciences \& Technology (2020): 10.5005/jp-journals-10057-0122
\end{abstract}

\section{INTRODUCTION}

Oral candidiasis is an opportunistic infection of the oral cavity. It is commonly diagnosed among elderly persons, specifically in those who wear dentures, and in many cases is preventable with a good mouth care regimen. It can also be a mark of systemic disease, e.g., diabetes mellitus, and is largely seen among immune-compromised patients. ${ }^{1}$ Oral candidiasis is caused by an excessive or infection of the oral cavity caused by a yeast-like fungus, Candida. ${ }^{2}$ Additional 20 species of Candida, Candida albicans, are the most collective and important causal agent of oral candidiasis. ${ }^{3}$ Candida albicans is a dimorphic fungal organism that characteristically is present in the oral cavity in a non-pathogenic state in about one-half of healthy persons. Normally present as yeast, the organism being under favorable environments, can transform into a pathogenic (diseasecausing) hyphae form. Some other Candida species are C. tropicalis, $C$. glabrata, C. pseudotropicalis, C. krusei, C. Iusitaniae, C. parapsilosis, and C. stellatoidea. ${ }^{3}$ The conditions that contribute to the development of the infection comprise broad-spectrum antibiotic treatment, xerostomia, immune dysfunction, or the presence of any removable prosthesis or denture. Many advancements in medical treatment, such as, antineoplastic chemotherapy, organ transplantation, hemodialysis, parenteral nutrition, and central venous catheters, are seen in contributing to fungal invasion and colonization. ${ }^{4}$

\section{Case Description}

A 56-year-old male patient came to the department with the chief complaint of loose pair of denture hence difficulty in eating food and wants replacement of new denture and also complaint of white patches on left posterior buccal mucosa which was developed for 5 months. The patient gives a history of wearing dentures for the last 6 years, he had undergone full mouth extraction 6 years ago due to multiple carious teeth and reveals that other family members also have the same dental problem, also gives a history of replacement of lower denture alone 6 months ago due to breakage of the denture while having food and had done replacement of lower denture alone on the same month since that time patient having ill-fitting denture and soon after he noticed developing white patches on the
${ }^{1-4}$ Department of Oral Medicine and Radiology, Mahatma Gandhi Dental College and Hospital, Mahatma Gandhi University of Medical Sciences and Technology, Jaipur, Rajasthan, India

Corresponding Author: Shimiyan Debbarma, Department of Oral Medicine and Radiology, Mahatma Gandhi Dental College and Hospital, Mahatma Gandhi University of Medical Sciences and Technology, Jaipur, Rajasthan, India, Phone: +91 8131903710, e-mail: shimiyandebbarma@gmail.com

How to cite this article: Debbarma S, Jamdade A, Yadav S, et al. Chronic Hyperplastic and Erythematous Candidiasis Induced by IIIfitting Complete Denture: A Case Report. J Mahatma Gandhi Univ Med Sci Tech 2020;5(1):31-33.

Source of support: Nil

Conflict of interest: None

left posterior buccal mucosa since 1 week which recently started having mild pain and burning sensation while having food as history given by the patient. The patient is unable to reveal whether it had developed as small size patches or any vesicle rupture and there was no history of blister eruption or cheek biting. The patient revealed that he had a habit to wear the denture for 24 hours and used to clean his denture using a toothbrush. The patient gave no significant past medical history. The patient had a history of chewing gutka 5 packets a day for the past many years about 20 years but had left the habit 6 years before soon after he started wearing a denture.

On intraoral examination, the patient is fully edentulous shown in Figure 1A. There is the existence of white and erythematous patches on left retrocommissural spaces spreading $2 \mathrm{~cm}$ posteriorly into the buccal mucosa and also $2 \mathrm{~cm}$ superiorly and inferiorly into the buccal mucosa. The erythematous part was covered with nodular, raised white projections shown in Figure 1B on palpation, the patches were non-scrapable and mild pain on touch, welldemarcated within the plaque. Misaligned of denture was noticed, crossbite of posterior teeth on left side (Fig. 1C). No extraoral oral finding was noted. A complete blood examination was advised on the first visit and a smear was prepared by scrapings of these areas of lesions for cytological evaluation, culture Candida was

(c) The Author(s). 2020 Open Access This article is distributed under the terms of the Creative Commons Attribution 4.0 International License (https:// creativecommons.org/licenses/by-nc/4.0/), which permits unrestricted use, distribution, and non-commercial reproduction in any medium, provided you give appropriate credit to the original author(s) and the source, provide a link to the Creative Commons license, and indicate if changes were made. The Creative Commons Public Domain Dedication waiver (http://creativecommons.org/publicdomain/zero/1.0/) applies to the data made available in this article, unless otherwise stated. 


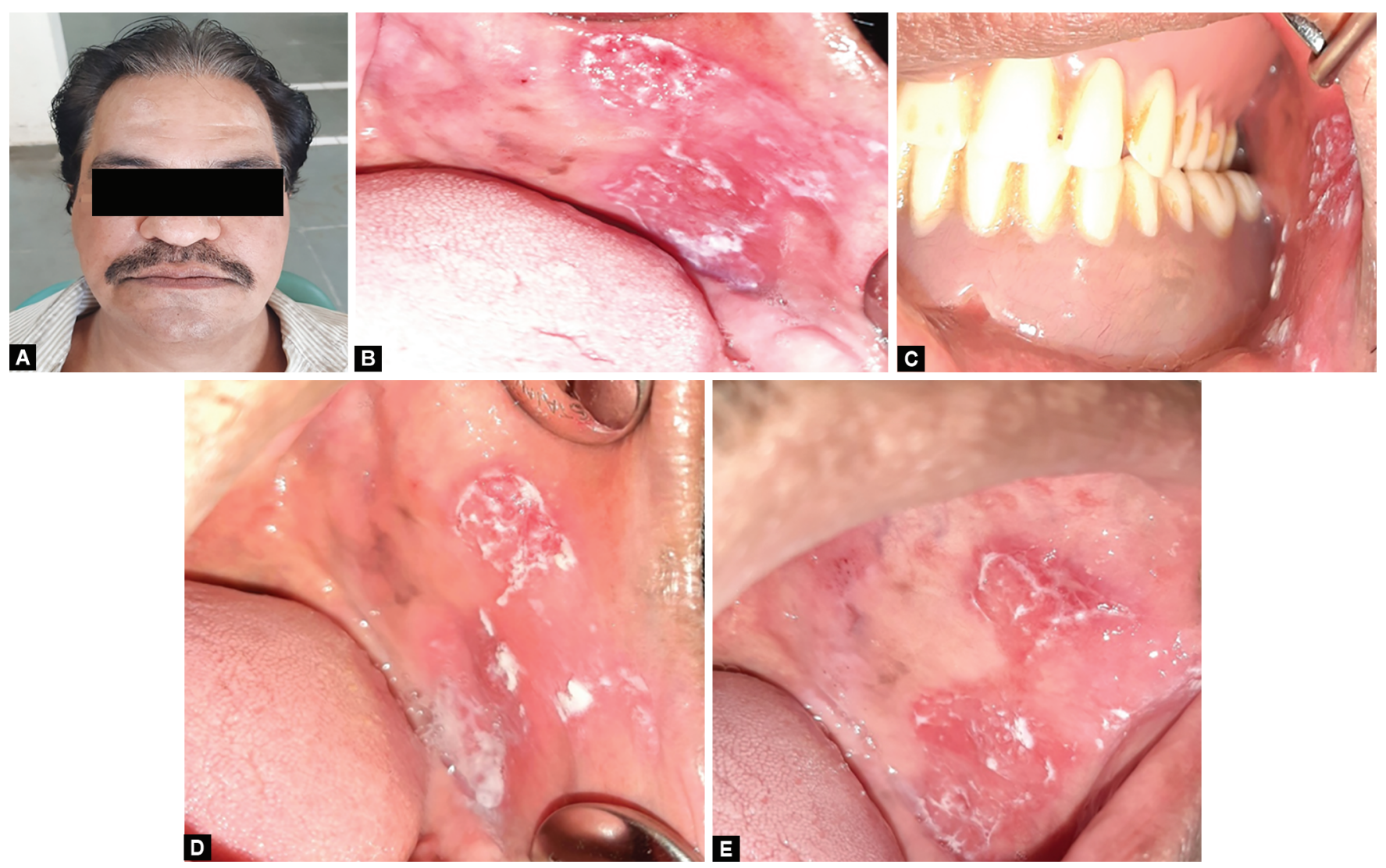

Figs 1 A to E: First visit: (A) Facial profile; (B) White erythematous patches on left retrocommissural area which is surrounded by a diffused reddish and elevated margin; (C) Left side posterior teeth are in a crossbite; Second visit: (D) Second visit after a week reduction in extension of white patches and erythematous margins compared to first visit; (E) Second visit after 2 weeks, reduction in white patches and elevated margins compared to previous visits

performed using a Sabouraud's dextrose agar and was also done to aid the ultimate identification of the fungal organism. Based on clinical features and history given by the patient, the provisional diagnosis was given as a mixed type of chronic hyperplastic and erythematous candidiasis induced by the ill-fitting denture. D/D Speckled leukoplakia, Lichen Planus.

The patient was treated with chlorhexidine mouth rinse twice a day, miconazole oral gel twice a day, tablet ketoconazole $200 \mathrm{mg}$ twice a day, vitamin C 100 mg twice a day, and vitamin B complex $100 \mathrm{mg}$ twice a day for 14 days. The patient was advised for new denture construction and instructed not to wear his denture until the new denture is made and to clean white pseudomembrane parts on his mouth using a soft toothbrush. Also advised for blood investigation to rule out any systemic underlying disease. On the follow-up visit, 1 week after an improvement of the presenting complaints was reported. Intraoral examination showed reducing/ disappearing in an extension of white and erythematous patch (Fig. 1D). The complete blood examination report showed normal figures and the cytological evaluation indicated candidiasis. On the same day, the primary impression was made by the prosthodontics department and the patient was instructed to continue the previously prescribed medication for one more week and revisit after a week. On a next follow-up visit, the second-week patient reported complete symptomatic relief, and also there was a reduction in a white patch and elevated margins compared with previous visits (Fig. 1E). The patient was instructed to stop the medication and maintain oral and denture hygiene and revisit after a week for follow-up.

\section{Discussion}

Prevalence, typical appearance, and ease of removal of the lesions marks oral thrush easily predictable, a diagnosis of thrush is often made based on the characteristic of the lesion. Taking a case history followed by a thorough clinical examination of the oral cavity, at the soft and hard palate, and examining the oral mucosa in those wearing dentures after they have been removed are generally good starting points. Diagnosis can be established microbiologically either by staining a smear from the affected area with periodic acid-Schiff (PAS) stain, Gridley stain, or Gomori methenamine silver (GMS) stain or by culture in gas wab Froman mouthwash. Culture Candida using a Sabouraud's agar slope was done to the identification of the fungal organism. This case was established based on clinical features, which are characterized by thrush that is white pseudomembrane erythematous base and with microbiology analysis using swab and culture Sabouraud's agar. Candida colony was present in microbiology examination.

Although C. albicans is the most commonly found species in oral candidiasis, other species are gradually being encountered. The unique virulence factors of $C$. albicans contain the capacity to adhere to host tissue surfaces, harvest filamentous fungal growth, and release hydrolytic enzymes that result in damage to the host tissue. Oral candidiasis is a state which arises due to some 
predisposing factors like different local resistance to infection, compromised immune system function, and generalized patient debilitation. Candida albicans that source the disease usually exists in the oral cavity in an inactive form. In most cases, the disease is often unnoticed and only proper oral examination by the dentist can diagnose it. Furthermore, it should be noted that every white lesion in the oral cavity is not due to the Candida infection. Although after the proper laboratory investigations, the disease can be confirmed. Diagnosis of oral candidiasis can be done by microscopic examination and/or biopsy. The disease can be easily controlled by administering topical or systemic antifungal agents. The prognosis of oral candidiasis is good. Candida albicans is found to be fundamental species in Candidaassociated denture stomatitis.

The occurrence of candidiasis in the oral cavity with predominant C. albicans isolation has been stated to be $45 \%$ in neonates, ${ }^{5} 45-65 \%$ in youngsters, ${ }^{6} 30-45 \%$ of healthy adults, ${ }^{7}$ $50-65 \%$ in cases of prolonged denture wearers, ${ }^{8} 65-88 \%$ in those residing in acute and long-term facilities, ${ }^{9-11} 90 \%$ in patients with acute leukemia undertaking chemotherapy, ${ }^{12}$ and $95 \%$ of patients with HIV infection. ${ }^{13}$ Systemic candidiasis conveys a mortality rate of $71-79 \%$. It is significant for all the clinicians treating the older patients to be alert of the risk factors, diagnosis, and dealing of oral candidiasis. In a recent study, it was shown that $30 \%$ of clinicians granted that, even without examining the oral cavity, they would recommend nystatin for oral candidiasis at the request of assistant staff. Such carelessness can result in a wrong diagnosis, missed pathologies, and failure to address the risk factors which may result in recurrence of candidiasis. ${ }^{14}$

\section{Conclusion}

This case concludes that mainly due to three factors these are reduced of host's immune position that was affected by patient's age (56years); oral mucosa situation that contributed to candidiasis, i.e., poor oral hygiene, nonstop denture wearing, and broken prosthesis of lower denture which may favor the presence $C$. albicans. In this case, oral candidiasis was established by complete clinical features which were also confirmed with microbiology investigation and treated with topical antifungals (miconazole oral gel) which were given in combined with a systemic antifungal (ketoconazole). The habit of wearing a nonstop denture for 24 hours and poor oral hygiene are predisposing factors of oral candidiasis. It can be cured completely with topical and systemic antifungal and by eliminating contributing factors. To avoid recurrence predisposing factors should be always found out and removed, then advise for replacement of denture if it is faulty, instructions to be given about proper oral hygiene maintenance followed by the use of a complete denture.

\section{References}

1. Epstein JB. Antifungal therapy in oropharyngeal mycotic infections. Oral Surg Oral Med Oral Pathol Oral Radiol 1990;69(1):32-41. DOI: 10.1016/0030-4220(90)90265-T.

2. Guida RA. Candidiasis of the oropharynx and oesophagus. Ear, Nose and Throat J 1988;67(11):832. 834-6, 838-840.

3. Premanathan M, Shakurfow FAA, Ismail AA, et al. Treatment of oral candidiasis (thrush) by Saccharo mycesce revisiae'. Int J Med Med Sci 2011;3(3):83-86.

4. Mikulska M, Del Bono V, Ratto S, et al. Occurrence, presentation and treatment of candidemia. Exp Rev Clini Immunol 2012;8(8):755-765. DOI: $10.1586 /$ eci.12.52.

5. Berdicevsky l, Ben-Aryeh $\mathrm{H}$, Szargel R, et al. Oral candida in children. Oral Surg Oral Med Oral Pathol Oral Radiol 1984;57(1):37-40. DOI: 10.1016/0030-4220(84)90257-3.

6. Lucas VS. Association of psychotropic drugs, prevalence of denture related stomatitis and oral candidosis. Commun Dent Oral Epidemiol 1993;21(5):313-316. DOI: 10.1111/j.1600-0528.1993.tb00782.x.

7. Arendorf TM, Walker DM. The prevalence and intra-oraldistribution of Candida albicans in man. Arch Oral Biol 1980;25(1):1-10. DOI: 10.1016/0003-9969(80)90147-8.

8. Aldred MJ, Addy M, Bagg J, et al. Oral health in the terminally ill: a cross-sectional pilot survey. Spec Care Dentist 1991;11(12):59-62.

9. Cumming CG, Wight C, Blackwell CL, et al. Denture stomatitis in the elderly. Molecul Oral Microbiol 1990;5(2):82-85.

10. Holbrook WP, Hjorleifsdottir DV. Occurrence of oral Candida albicans and other yeast-like fungi in edentulous patients in geriatric units in Iceland. Gerodontics 1986;2(5):153-156.

11. Rodu B, Carpenter JT, Jones MR. The pathogenesis and clinical significance of cytologically detectable oral Candida in acute leukemia. Cancer 1988;62(9):20422046. DOI: 10.1002/1097-0142(19881101)62:9<2042::AIDCNCR2820620928>3.0.CO;2-D.

12. Dupont B, Graybill JR, Armstrong D, et al. Fungal infections in AIDS patients. J Med Vet Mycol 1992;30(1):19-28. DOI: 10.1080/02681219280000731.

13. Fraser VJ, Jones M, Dunkel J, et al. Candidemia in a tertiary care hospital: epidemiology, risk factors, and predictors of mortality. Clin Infect Dis 1992;15(3):414-421. DOI: 10.1093/clind/15.3.414.

14. Rathod P, Punga R, Dalal V, et al. Oral candidiasis widely prevalent. Frequen Miss Int J Scienti Stu 2015;3(6):193-198. 